\title{
Pharmacokinetics of
}

\section{combined treatment with}

\section{praziquantel and \\ albendazole in}

\section{neurocysticercosis}

Hector H. Garcia, ${ }^{1,2}$ Andres G. Lescano, ${ }^{2,3}$ Vera L. Lanchote, ${ }^{4}$

E. Javier Pretell, ${ }^{1,2}$ Isidro Gonzales, ${ }^{1}$ Javier A. Bustos, ${ }^{2}$

Osvaldo M. Takayanagui, ${ }^{5}$ Pierina S. Bonato, ${ }^{4}$ John Horton, ${ }^{6}$

Herbert Saavedra, ${ }^{1}$ Armando E. Gonzalez ${ }^{7} \&$ Robert H. Gilman ${ }^{2,8}$ for the Cysticercosis Working Group in Peru

${ }^{1}$ Cysticercosis Unit, Department of Transmissible Diseases, Instituto Nacional de Ciencias Neurologicas, Jr. Ancash 1271, Barrios Altos, Lima 1, 2Department of Microbiology, School of Sciences (HHG), and School of Public Health (AGL, JAB) Universidad Peruana Cayetano Heredia, Av. H. Delgado 430, SMP, Lima 31, ${ }^{3}$ Naval Medical Research Centre Detachment, Av. Venezuela c.36 s/ $n$, Bellavista, Callao, Peru, ${ }^{4}$ Faculdade de Ciências Farmacêuticas de Ribeirão Preto, Universidade de São Paulo, Avenida do Café s/n, Campus da USP, 14040-903, Ribeirão Preto, SP, ${ }^{5}$ Faculdade de Medicina de Ribeirão Preto, Universidade de São Paulo,Avenida do Café s/n, Campus da USP, 14040-903, Ribeirão Preto, SP, Brazil, ${ }^{6}$ Tropical Projects, 24 Paddock, Hitchin, Herts SG4 9EF, UK, ${ }^{7}$ School of Veterinary Medicine, Universidad Nacional Mayor de San Marcos, Av. Circunvalacion s/n, Salamanca de Monterrico, Ate, Lima, Perú and ${ }^{8}$ Department of International Health, Johns Hopkins Bloomberg School of Public Health, 615 N. Wolfe Street, Baltimore, MD 21205, USA

\section{Correspondence}

Prof. Hector H. Garcia MD PhD,

Department of Microbiology, School of Sciences and Centre for Global Health, Universidad Peruana Cayetano Heredia, $\mathrm{H}$. Delgado 430, SMP, Lima 31, Perú.

Tel.: + 5113287360

Fax: + 5113284038

E-mail: hgarcia@jhsph.edu

Keywords

albendazole, neurocysticercosis, Peru, praziquantel, Taenia solium

Received

23 April 2010

Accepted

1 January 2011

Accepted Article

18 February 2011

\section{WHAT IS ALREADY KNOWN ABOUT} THIS SUBJECT

- The antiparasitic drugs albendazole and prazoquantel have been used together before for geohelminths, echinococcosis and cysticercosis, but there is only scarce and discrepant data on the pharmacokinetics of these drugs when given together.

\section{WHAT THIS STUDY ADDS}

-This is a randomized, double-blinded, placebo-controlled phase II evaluation of the pharmacokinetics of albendazole and praziquantel in 32 patients with neurocysticercosis which demonstrates that combined albendazole + praziquantel is associated with increased albendazole sulfoxide concentrations. These increased concentrations could independently contribute to increased cysticidal efficacy by themselves or in addition to a possible synergistic effect.

\section{AIMS}

Neurocysticercosis is the most common cause of acquired epilepsy in the world. Antiparasitic treatment of viable brain cysts is of clinical benefit, but current antiparasitic regimes provide incomplete parasiticidal efficacy. Combined use of two antiparasitic drugs may improve clearance of brain parasites. Albendazole (ABZ) has been used together with praziquantel (PZQ) before for geohelminths, echinococcosis and cysticercosis, but their combined use is not yet formally recommended and only scarce, discrepant data exist on their pharmacokinetics when given together. We assessed the pharmacokinetics of their combined use for the treatment of neurocysticercosis.

\section{METHODS}

A randomized, double-blind, placebo-controlled phase II evaluation of the pharmacokinetics of $A B Z$ and $P Z Q$ in 32 patients with neurocysticercosis was carried out. Patients received their usual concomitant medications including an antiepileptic drug, dexamethasone, and ranitidine. Randomization was stratified by antiepileptic drug (phenytoin or carbamazepine). Subjects had sequential blood samples taken after the first dose of antiparasitic drugs and again after 9 days of treatment, and were followed for 3 months after dosing.

\section{RESULTS}

Twenty-one men and 11 women, aged 16 to 55 (mean age 28) years were included. Albendazole sulfoxide concentrations were increased in the combination group compared with the $A B Z$ alone group, both in patients taking phenytoin and patients taking carbamazepine. PZQ concentrations were also increased by the end of therapy. There were no significant side effects in this study group.

\section{CONCLUSIONS}

Combined $\mathrm{ABZ}+\mathrm{PZQ}$ is associated with increased albendazole sulfoxide plasma concentrations. These increased concentrations could independently contribute to increased cysticidal efficacy by themselves or in addition to a possible synergistic effect. 


\section{Introduction}

Neurocysticercosis (NCC) is the most common cause of acquired epilepsy in the world [1]. Antiparasitic treatment of neurocysticercosis uses either albendazole (ABZ) or praziquantel (PZQ). Despite a long controversy in the literature, it is currently accepted that antiparasitic treatment and subsequent death of viable parenchymal brain cysts improves the prognosis of the associated seizure disorder $[2,3]$. Cyst death is not a direct drug effect but actually results from the host's immune system destroying the parasite after antigenic exposure caused by treatmentassociated damage. Failure of therapy to eradicate viable lesions remains a problem, because either $A B Z$ or $P Z Q$ have only partial cysticidal efficacy [4]. ABZ and PZQ have different mechanisms of action on the parasitic cyst. It is biologically plausible that their combined use will improve clearance of viable brain cysts and thus provide better cysticidal efficacy without increased risks. Such combination therapy offers the potential benefit of enhanced seizure control over single-agent therapy and would have worldwide applicability.

A single combined dose of ABZ (400 mg) and PZQ $(600 \mathrm{mg})$ has been used for a long time in geohelminth control campaigns. Three studies have evaluated their pharmacokinetics. In the first, Homeida et al. [5] found, in healthy volunteers given a $400-\mathrm{mg}$ dose of $A B Z$, a 4.5 -fold increase in the AUC of albendazole sulfoxide (ABSO), the active metabolites of $A B Z$, when administered simultaneously with PZQ (40 mg kg-1), an increase of eightfold when given with food and of 12 -fold when given with $P Z Q$ and food. Concentrations of PZQ were not significantly affected by the administration of ABZ. Ten years later, Pengsaa et al. [6] examined the interaction in Thai children with giardiasis given $400 \mathrm{mg}$ of $A B Z$ and $20 \mathrm{mg} \mathrm{kg}^{-1}$ of PZQ without finding significant interactions. More recently, Na-Bangchang et al. [7] used different single-dose regimes including $A B Z, A B Z+P Z Q+$ ivermectin and $P Z Q+$ ivermectin. They found minor changes in the kinetics of $A B S O$, not considered to be of clinical relevance. The combination $A B Z+P Z Q$ is widely used for presurgical prophylaxis and treatment of human echinococcosis at a dose of 400$800 \mathrm{mg}^{\text {day }}{ }^{-1}$ of $A B Z$ and 25 to $50 \mathrm{mg} \mathrm{kg}^{-1}$ day $^{-1}$ of PZQ, usually for multiple periods of 2 weeks with a 1-week break [8-12]. It has also been used for larva migrans [13] and clonorchiasis [14], and more recently in cysticercosis [15, 16]. Pharmacokinetic data on multiple dose combination regimes are not yet available.

Antiparasitic treatment of NCC is administered with a number of concomitant medications including steroids, $\mathrm{H}_{2}$-receptor blockers and antiepileptic drugs (AEDs), all of which can affect the pharmacokinetics of both drugs. We performed a double-blind, placebo-controlled, randomized trial in 32 patients with NCC to measure and compare plasma concentrations of $\mathrm{PZQ}$ and $\mathrm{ABSO}$ (the main metabolite of $A B Z)$.

\section{Methods}

Study design

This was a randomized, double-blind, placebo-controlled phase II evaluation of the pharmacokinetics of 9.5-day regimes of $A B Z$ with and without PZQ in patients with NCC. All patients received usual concomitant medications including an AED, dexamethasone, and ranitidine (Figure 1).

\section{Population}

Patients were enrolled at the Cysticercosis Unit, Instituto Nacional de Ciencias Neurologicas (INCN), Lima, Peru. The INCN is the national reference centre for neurology in Peru. The study enrolled male or female individuals between 16 and 65 years of age, with a diagnosis of NCC and 20 or fewer viable cysts as demonstrated by MRI and CT scan, confirmed by serology on Western blot and with a diagnosis of epilepsy secondary to NCC. Other inclusion criteria included willingness to complete a minimum of 2 weeks of hospitalization, use of an adequate method of contraception while on study medications and for at least 1 month following $A B Z$ therapy, normal laboratory values for haematocrit, platelets, WBC and glucose, AST, ALT and creatinine; negative purified protein derivative measurement or if positive ( $>9 \mathrm{~mm}$ induration in the absence of other findings or immunosuppression), negative smears for TB and negative faecal examinations for Taenia eggs or Strongyloides larvae. By the time of randomization, patients should have had been receiving a stable monotherapy regime of phenytoin or carbamazepine for at least 15 days. The screening phase included a complete history and physical examination including eye fundus examination, laboratory testing, EEG and brain CT and MRI scans.

Exclusion criteria included primary generalized seizures, history of generalized status epilepticus, types of NCC which could expose the patient to increased risk during the study, specifically (i) subarachnoid cysts in the Sylvian fissure or basal cisterns excepting Sylvian fissure located cysts substantially surrounded by brain parenchyma by MRI; (ii) intraventricular cysts; (iii) cysts in brainstem; (iv) any vesicular lesion greater than $2.5 \mathrm{~cm}$ of diameter; or (v) untreated ocular cysticercosis; persistent or progressive symptomatic intracranial hypertension $(\mathrm{ICH})$ or $\mathrm{ICH}$ defined radiologically, previous therapy with $\mathrm{ABZ}$ or PZQ in the past 2 years, except for patients treated with up to $1200 \mathrm{mg}$ of ABZ 2 months or greater prior to evaluation who demonstrated continued viability of cysts, pulmonary tuberculosis evidenced by a positive chest $\mathrm{X}$-ray and positive sputum smears, or symptoms compatible with tuberculosis (fever + sweats or fever + cough) not otherwise explained, positive markers for active hepatitis, systemic disease other than NCC, unstable condition, hypersensitivity to $\mathrm{ABZ}$ or $\mathrm{PZQ}$, concurrent treatment with cimetidine or theophylline, chronic alcohol or drug abuse, inability or unwillingness to have follow-up CT scan or MRI examina- 


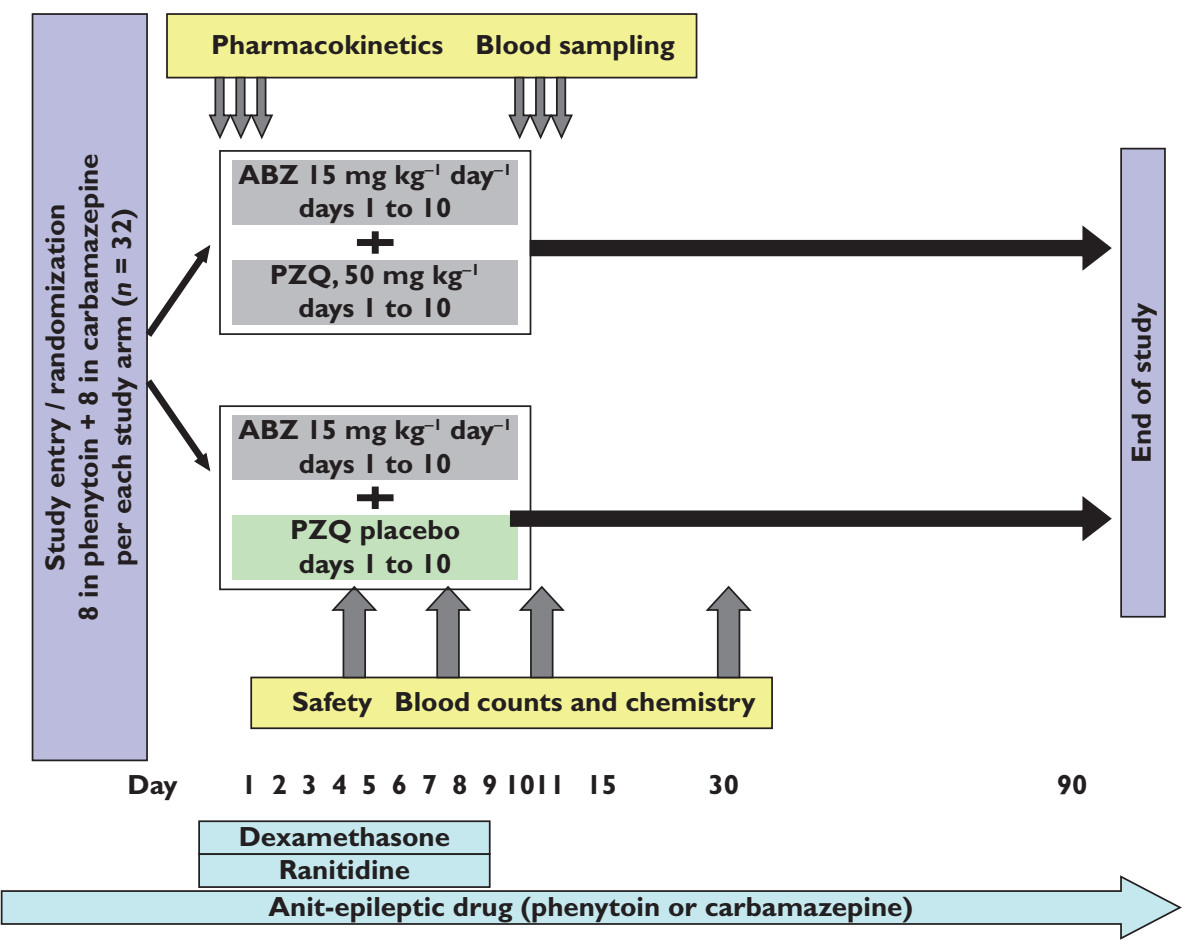

\section{Figure 1}

Study flowchart. ABZ, albendazole; PZQ, praziquantel

tion or inability or unwillingness of subject or legal guardian/representative to give written informed consent.

Patients were asked not to drink caffeine-containing beverages and to refrain from alcohol intake for at least 2 days before and after taking the study medication. None of the patients used known inducers or inhibitors of CYP enzymes other than phenytoin or carbamazepine for at least 2 weeks before entry into the study.

\section{Randomization}

Praziquantel in 600-mg tablets, similarly appearing PZQ placebo and $A B Z$ in 200-mg tablets were purchased from commercial sources. $\mathrm{PZQ} /$ placebo randomization was performed using a predetermined randomization procedure, using a mixture (also randomly generated) of blocks of size 4 and 6 . Randomization was stratified by AED (carbamazepine/phenytoin).

The medications were provided to the study physician in sealed, labelled envelopes, one for $\mathrm{PZQ}$ or placebo (containing $P Z Q$ in 16 cases and placebo in the remaining 16) and one for $A B Z$ (containing active drug for all patients).

\section{Intervention and comparators}

Intervention Praziquantel $\left(50 \mathrm{mg} \mathrm{kg}^{-1}\right.$ day $^{-1}$, up to $3600 \mathrm{mg} \mathrm{day}^{-1}$, for 9.5 days), as an add-on to $A B Z$ treatment (15 $\mathrm{mg} \mathrm{kg}^{-1}$ day $^{-1}$, up to $800 \mathrm{mg} \mathrm{day}^{-1}$, for 9.5 days).
Comparison regimen Praziquantel placebo, given during ABZ treatment $\left(15 \mathrm{mg} \mathrm{kg}^{-1}\right.$ day $^{-1}$, up to $800 \mathrm{mg} \mathrm{day}^{-1}$, for 9.5 days).

\section{Administration and duration}

Study subjects received approximately $50 \mathrm{mg} \mathrm{kg}^{-1}$ day $^{-1}$ of PZQ or PZQ placebo divided in two daily doses, morning and evening. PZQ comes in 600-mg tablets divided in four, so the dose was rounded up to the next 150-mg level, up to a maximum of $3.6 \mathrm{~g} \mathrm{day}^{-1}$. $\mathrm{ABZ}$ was given at $15 \mathrm{mg} \mathrm{kg}^{-1}$ day $^{-1}$ divided in two daily doses, morning and evening. $A B Z$ is supplied in 200-mg tablets so the dose was rounded up to the next $100-\mathrm{mg}$ level, up to a maximum of $800 \mathrm{mg}$ day $^{-1}$. Drugs were administered in the hospital, by the study nurse.

The absorption of orally administered ABZ is profoundly influenced by co-administration with food, particularly fatty food $[17,18]$. Although the mechanism remains to be demonstrated, diets with a high lipid or carbohydrate content also increase PZQ bioavailability in healthy volunteers [19]. Patients in this study received a hospital diet which is standardized in number of calories and not enriched in fat. The initial and last doses of antiparasitic medication were administered after fasting for $12 \mathrm{~h}$ before and fasting was continued for $4 \mathrm{~h}$ after administration.

\section{Concomitant medications}

Patients should have been started on appropriate and stable doses of phenytoin or carbamazepine at least 
15 days before randomization. One day prior to initiation of antiparasitic therapy, patients were started on dexamethasone at $0.1 \mathrm{mg} \mathrm{kg}^{-1}$ day $^{-1}$, divided in two doses (morning and evening) unless higher doses were needed to control intracranial hypertension. Additionally, to prevent gastrointestinal symptoms caused by the dexamethasone, patients in both groups received $300 \mathrm{mg} \mathrm{day}^{-1}$ of ranitidine.

\section{Blood sampling for drug concentrations}

Before any study medication was given, blood was drawn for baseline safety data as detailed below. In the morning of the first treatment day, a heparin lock was inserted, and 3-ml blood samples for ABSO and PZQ concentrations were drawn at $0,0.5,1,1.5,2,3,4,8,10$ and $12 \mathrm{~h}$ after the first dose. At that point, the heparin lock was removed and reinserted on treatment day 10 to collect blood samples for ABSO and PZQ concentrations at 0.5, 1, 1.5, 2, 3, 4, 8, 10, 12,24 and $36 \mathrm{~h}$ after the last dose of antiparasitic treatment (before discharge from hospital). Immediately after collection, blood samples were centrifuged at $1200 \mathrm{~g}$ for $15 \mathrm{~min}$ and plasma samples were separated and stored at $-80^{\circ} \mathrm{C}$ until analysis.

\section{Simultaneous determination of albendazole sulfoxide and praziquantel in plasma}

Plasma samples were analysed by a previously validated analytical method using LC-MS/MS [20]. The plasma samples $(1 \mathrm{ml})$ spiked with phenacetin as internal standard were prepared by liquid-liquid extraction and $A B S O$ and PZQ were separated on a Lichrospher CN column using methanol: water $(3: 7, \mathrm{v}: \mathrm{v})$ plus $0.5 \%$ of acetic acid as mobile phase. Quantification was performed by multiple reaction monitoring of the protonated molecules and their corresponding production [ABSO (282 > 240), PZQ (313> 203) and internal standard $(180>110]$. The lower limit of quantification was $5 \mathrm{ng} \mathrm{ml}^{-1}$ and the linear range was $5-2500 \mathrm{ng} \mathrm{ml}^{-1}$ for all analytes. The coefficients of variation and the relative errors obtained for the validation of intraand interassay precision and accuracy were less than $15 \%$. ABSO (purity 99.4\%) was kindly supplied by Robert Young \& Co. Ltd (Glasgow, Scotland, UK) and PZQ (purity > 99\%) was kindly supplied by Merck (Rio de Janeiro, Brazil). All chemicals of analytical grade were purchased from Merck (Darmstadt, Germany) or EM Science (Gibbstown, NJ, USA).

\section{Pharmacokinetic analysis}

The pharmacokinetic parameters $t_{\max }, C_{\max }, A \cup C(0,4 \mathrm{~h})$ for the initial dose, and $A \cup C(0,12 \mathrm{~h})$ for the last dose for $A B S O$ and PZQ were calculated based on the plasma concentration vs. time curves using the WinNonlin software, version 4.0 (Pharsight Corp. Mountain View, CA, USA). The calculations were performed using first-order kinetics and a monocompartmental model with and without the inclusion of lag time.

\section{Statistical analysis}

Statistical analyses were carried out with Graphpad Instat software in order to obtain the mean, median and $95 \% \mathrm{Cl}$. The Mann-Whitney test $(P<0.05)$ was used to evaluate the effect of PZQ on $A B Z$ metabolism or the effect of $A B Z$ on the kinetic disposition of PZQ.

\section{Blood sampling and safety monitoring}

Haemoglobin, haematocrit, platelet counts, total white blood cell and differential counts, creatinine, glucose, bilirubin, ALT, AST and electrolytes were monitored at screening and days 4, 7, 11 and 30 of antiparasitic treatment. Safety monitoring and adverse event recording were continued for 3 months. The study was completed 3 months after last enrollment (August 2007-September 2008).

\section{Human subjects protection}

The study protocol and informed consents were revised and approved by the main IRB of the Universidad Peruana Cayetano Heredia in Lima, Peru (IRB Code 51070, FWA 00002541). A Data Safety Monitoring Board was established prior to commencing the programme and supervised the entire study. There was no direct monetary compensation to the subjects. In exceptional cases, patients at high risk of being lost to follow-up were compensated for the time lost to attend a follow-up visit or examination.

\section{Results}

Twenty-one men and 11 women, with a mean age of 28 years, were enrolled. Treatment groups were clearly similar in terms of age, gender, height and weight (Table 1). Sample volume was insufficient to complete the testing for ABSO concentrations in the initial dose in two cases and in the final dose in one case.

\section{Albendazole sulfoxide concentrations with and without praziquantel}

The concentrations of the active metabolite of $A B Z, A B S O$, were not significantly different between groups after the first dose of antiparasitic treatment. After the last dose, however, both $C_{\max }$ and the AUC for ABSO were signifi-

\section{Table 1}

Demographic data of clinical study participants

\begin{tabular}{|lccc|}
\hline Group & $\begin{array}{l}\text { Albendazole }+ \\
\text { placebo }(n=16)\end{array}$ & $\begin{array}{l}\text { Albendazole }+ \\
\text { praziquantel }(n=16)\end{array}$ & $\boldsymbol{P}$ \\
\hline Gender (M/F) & $10(62.5 \%)$ & $11(68.8 \%)$ & NS \\
\hline Age (years, mean \pm SD) & $27.5 \pm 11.0$ & $29.3 \pm 9.7$ & NS \\
Weight (kg, mean \pm SD) & $61.9 \pm 11.7$ & $61.0 \pm 12.6$ & NS \\
\hline Height (cm, mean \pm SD) & $160.9 \pm 7.6$ & $161.0 \pm 7.9$ & NS \\
\hline
\end{tabular}


Table 2

Albendazole sulfoxide pharmacokinetic parameters by study group after the first and last dose

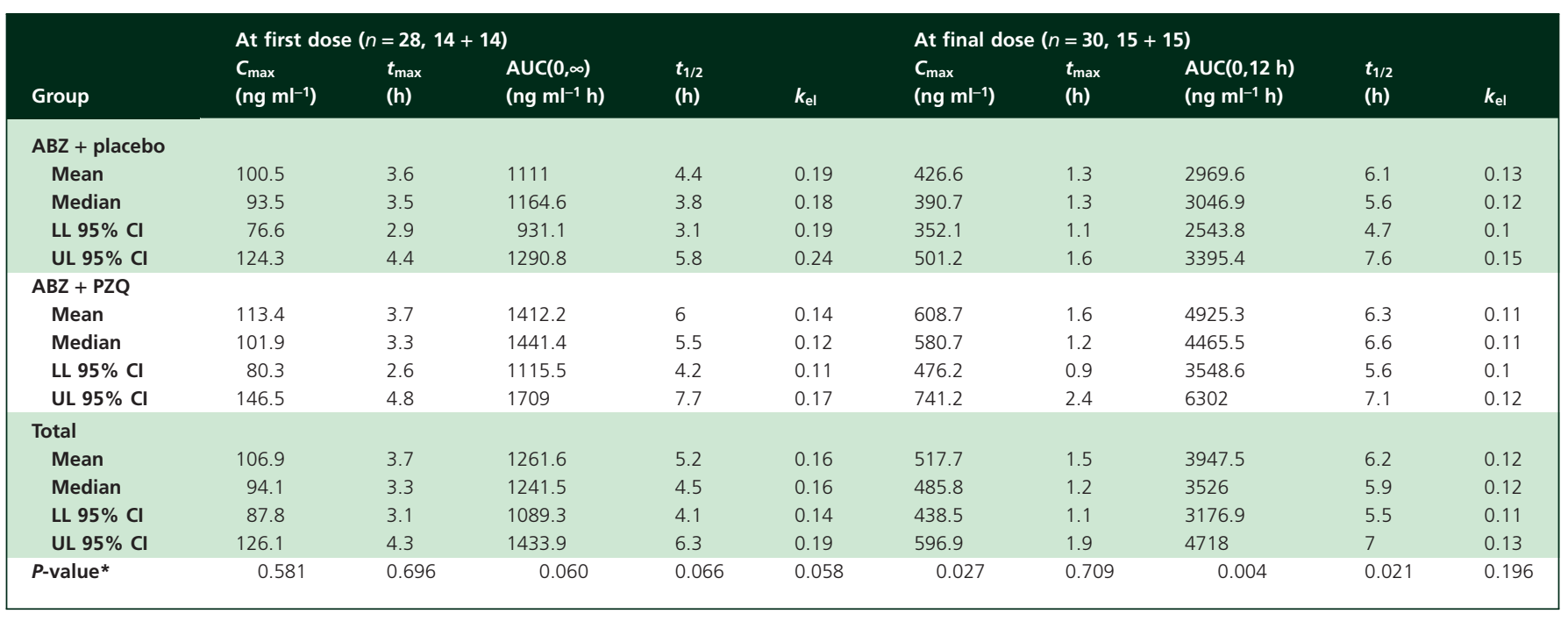

*Wilcoxon Mann-Whitney non-parametric rank sum test. ABZ, albendazole; PZQ, praziquantel.

cantly increased by approximately $50 \%$ in patients receiving PZQ compared with those receiving placebo (Table 2). There was a clear decrease in $t_{\max }$ between the first and last doses.

\section{Effect of phenytoin and carbamazepine on albendazole sulfoxide concentrations}

There were no significant differences in the concentrations of ABSO between patients receiving phenytoin or carbamazepine. The increase in concentrations of ABSO associated with the addition of PZQ was present in both patients receiving phenytoin or carbamazepine (Table 3 ).

\section{Praziquantel concentrations}

Concentrations of PZQ were measured in 16 patients, all of them simultaneously receiving ABZ. Maximum plasma concentrations of PZQ increased after the last dose compared with the concentrations taken after the first dose, both in patients receiving carbamazepine (21.9 to $54.0 \mathrm{ng} \mathrm{ml}^{-1}$, median values $n=8$ ) or phenytoin (34.9 to $245.0 \mathrm{ng} \mathrm{ml}^{-1}$, median values $n=8$ ). Serum concentrations of PZQ increased after the last dose compared with those after the first dose, both in patients receiving carbamazepine (median 21.9 to $54.0 \mathrm{ng} \mathrm{ml}^{-1}, 2.4$-fold, $n=8$ ) or phenytoin (median 34.9 to 245.0 , sevenfold, $n=8$ ).

\section{Effect of phenytoin and carbamazepine on $P Z Q$ concentrations}

After the last PZQ dose both $C_{\max }$ and AUC were significantly higher in the group receiving phenytoin compared with the group receiving carbamazepine (Table 4).

\section{Side effects}

There were no serious side effects in the entire group, and there were no significant differences in haematological or biochemical profiles between the 16 patients receiving PZQ compared with the 16 patients receiving placebo.

\section{Discussion}

This phase II trial comparing combined $A B Z+P Z Q$ to $A B Z$ plus placebo treatment in patients with NCC, with concomitant steroid and antiepileptic medication demonstrated a significant increase in ABSO concentrations in the combined therapy group after a 9.5-day treatment regime. In view of the results, if parasiticidal efficacy of the combination is shown to be higher than standard ABZ therapy, it may be because of a true synergistic effect of both drugs or to increased concentrations of the active ABZ metabolite. A phase III study is now ongoing to assess parasiticidal efficacy, including a comparison group with a higher dose of $A B Z$ to answer the above question. Of interest, $A B Z$ and PZQ concentrations were increased in both groups by the end of therapy, suggesting that multiple-dose regimens may be associated with better parasiticidal effects. The study was not powered to demonstrate parasiticidal efficacy and thus these results are not presented here. While this study did not show any side effects or raise any safety issues, the small numbers of patients studied make this safety assessment very preliminary.

After the initial dose, the plasma concentrations of ABSO increased only slightly in patients receiving PZQ compared with those receiving placebo. However, by the end of therapy, the plasma concentrations of ABSO were over $50 \%$ higher in the combined treatment group. An increase in ABSO concentrations may be because of increased rates of $A B Z$ oxidation, or decreased rates of albendazole sulphone (ASON) formation. Probably CYP 


\section{Table 3}

Albendazole sulfoxide pharmacokinetic parameters by antiepileptic drug and study group after the first and last dose

\begin{tabular}{|c|c|c|c|c|c|c|c|c|c|c|}
\hline \multirow[b]{2}{*}{ Group } & \multicolumn{5}{|c|}{ At first dose $(n=28,6+8+7+7)$} & \multicolumn{5}{|c|}{ At final dose $(n=30,7+8+7+8)$} \\
\hline & $\begin{array}{l}C_{\max } \\
\left(\mathrm{ng} \mathrm{ml}^{-1}\right)\end{array}$ & $\begin{array}{l}t_{\max } \\
\text { (h) }\end{array}$ & $\begin{array}{l}\operatorname{AUC}(0, \infty) \\
\left(n g \mathrm{l}^{-1} \mathrm{~h}\right)\end{array}$ & $\begin{array}{l}t_{1 / 2} \\
\text { (h) }\end{array}$ & $k_{\mathrm{el}}$ & $\begin{array}{l}C_{\max } \\
\left(\mathrm{ng} \mathrm{ml}^{-1}\right)\end{array}$ & $\begin{array}{l}t_{\max } \\
\text { (h) }\end{array}$ & $\begin{array}{l}\operatorname{AUC}(0,12 \mathrm{~h}) \\
\text { (ng ml-1 h) }\end{array}$ & $\begin{array}{l}t_{1 / 2} \\
\text { (h) }\end{array}$ & $k_{\mathrm{el}}$ \\
\hline \multicolumn{11}{|l|}{ ABZ + Placeb } \\
\hline Mean & 104.2 & 4 & 1217 & 4.4 & 0.19 & 444.1 & 1.1 & 3269.9 & 6.5 & 0.11 \\
\hline Median & 95.6 & 3.6 & 1261.4 & 4.1 & 0.17 & 485.9 & 1 & 3388.3 & 6 & 0.11 \\
\hline LL $95 \%$ Cl & 59.8 & 2.3 & 839.3 & 2.3 & 0.1 & 377 & 0.8 & 2931.3 & 4.8 & 0.08 \\
\hline Mean & 97.7 & 3.4 & 1031.5 & 4.4 & 0.2 & 411.4 & 1.5 & 2706.9 & 5.8 & 0.14 \\
\hline Median & 90.9 & 3.4 & 1114.9 & 3.6 & 0.19 & 370.5 & 1.4 & 2553.3 & 4.9 & 0.15 \\
\hline LL $95 \%$ Cl & 61.5 & 2.5 & 809 & 2.1 & 0.13 & 263.7 & 1.1 & 1914.5 & 3.1 & 0.1 \\
\hline UL $95 \% \mathrm{CI}$ & 133.8 & 4.3 & 1254 & 6.7 & 0.26 & 559 & 2 & 3499.4 & 8.5 & 0.18 \\
\hline$P$-value* & 0.796 & 0.606 & 0.245 & 0.606 & 0.746 & 0.105 & 0.064 & 0.133 & 0.083 & 0.102 \\
\hline \multicolumn{11}{|l|}{$A B Z+P Z Q$} \\
\hline \multicolumn{11}{|l|}{ Phenytoin } \\
\hline Mean & 113.2 & 4.1 & 1537.1 & 7.1 & 0.11 & 642.1 & 1.2 & 4909.2 & 6.9 & 0.1 \\
\hline Median & 92.9 & 3.6 & 1436.8 & 5.6 & 0.12 & 576.9 & 1.2 & 4446.2 & 7 & 0.1 \\
\hline LL $95 \%$ Cl & 55.9 & 2.2 & 1133.1 & 3.6 & 0.08 & 474.3 & 1 & 3822.6 & 5.7 & 0.09 \\
\hline UL $95 \%$ CI & 170.5 & 6.1 & 1941 & 10.5 & 0.15 & 809.9 & 1.4 & 5995.8 & 8.1 & 0.12 \\
\hline$P$-value* & 0.949 & 0.338 & 0.749 & 0.225 & 0.177 & 0.729 & 0.105 & 0.729 & 0.073 & 0.090 \\
\hline
\end{tabular}

*Wilcoxon Mann-Whitney non-parametric rank sum test. ABZ, albendazole; PZQ, praziquantel.

\section{Table 4}

Praziquantel pharmacokinetic parameters by antiepileptic drug after the first and last dose

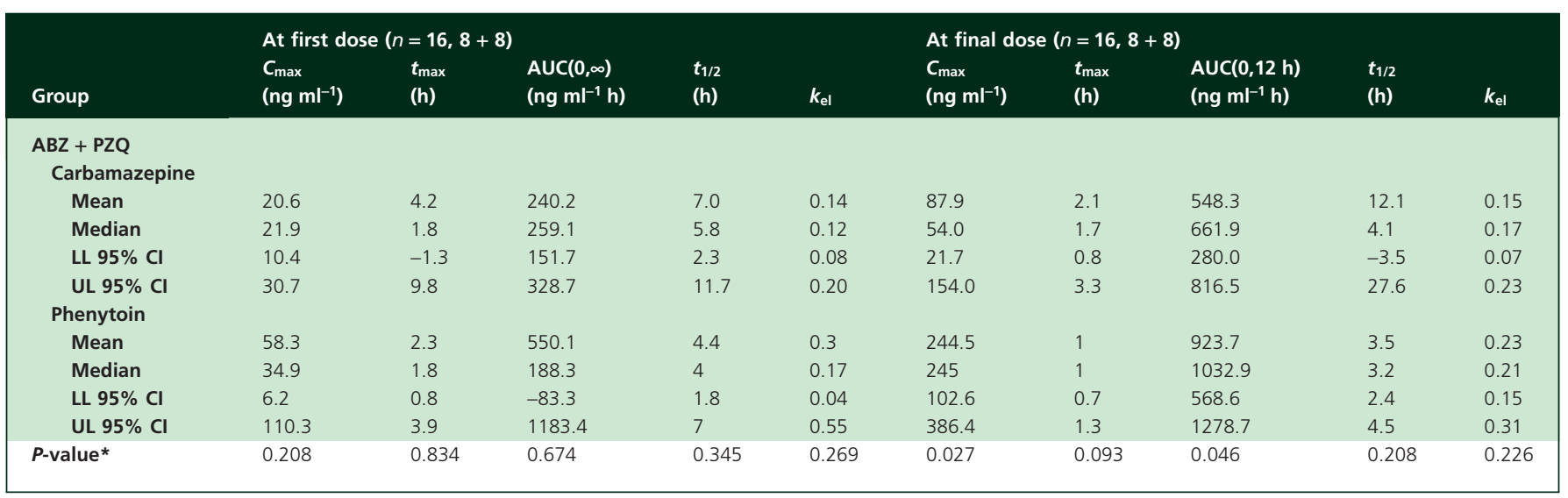

*Wilcoxon Mann-Whitney non-parametric rank sum test. ABZ, albendazole; PZQ, praziquantel.

enzymes involved in $A B Z$ oxidation (CYP3A4 as the major contributor) are not the same as involved in ASON formation (not yet known which CYP isoforms) [21]. As both $A B S O$ and $A S O N$ increased over time in patients receiving combined therapy (data not shown), the differential increase after combined therapy is more likely because of increased ABZ oxidation [22]. The increase in ABSO concentrations when used in combination with $\mathrm{PZQ}$ occurred both in presence of phenytoin or carbamazepine. There were only small differences in concentrations of $A B S O$ between patients taking carbamazepine or phenytoin.

Albendazole is a benzimidazole which acts primarily through selective degeneration of parasite cytoplasmic microtubules, leading to decreased ATP formation and energy depletion. It also has antimitotic activity (binds to tubulin), disrupting cell division, and causes loss of trans- 
port of secretory vesicles alter glucose intake, leading to parasite starvation. Peak plasma concentrations of the active metabolite ABSO occur 2-3 h after administration. It has very good penetration in CSF ( $43 \%$ of plasma concentrations). In human liver microsomes $A B Z$ is rapidly oxidized to $A B S O$, probably responsible for the therapeutic effect, apparently via both FMO3 and CYP3A4, with CYP being the major contributor [21]. ABSO is subsequently sulfoned to ASON, considered to be pharmacologically inactive [23]. No data regarding CYP isoforms involved in the sulfonation of ABSO in humans are available. PZQ is a pyrazinoisoquinoline derivative whose primary effects include muscle contractions, paralysis and tegumentary damage. Other effects include changes in carbohydrate metabolism, decrease in enzymatic activities and alterations in surface membranes. Peak plasma concentrations occur 1.5 to $2 \mathrm{~h}$ after administration. PZQ penetrates well into CSF, although less than ABZ (24\% of plasma concentration at steady state). $\mathrm{PZQ}$ is rapidly metabolized to inactive metabolites by CYPs, particularly CYP2B1 and CYP3A4. The metabolite found at a higher proportion in human plasma is the non-active trans-4-hydroxypraziquantel [24]. Bioavailability of PZQ has been shown to be reduced by inducers of hepatic CYP pathways $[25,26]$.

Albendazole sulfoxide concentrations are highly variable between patients. A recent prospective study performed in 90 patients with NCC receiving $30 \mathrm{mg} \mathrm{kg}^{-1}$ day $^{-1}$ of $A B Z$ for 8 days showed considerable variability in ABSO plasma concentrations because of drug bioavailability ranging $28-100 \%$ [27].The low aqueous solubility of $A B Z$ is probably the major determinant of its bioavailability. In addition to this underlying variability, antiparasitic tratment in NCC is administered concomitantly with other drugs, such as AEDs (carbamazepine or phenytoin in this trial), dexamethasone and ranitidine. Both carbamazepine and phenytoin stimulate the activity of a variety of CYP enzymes, including CYP1A2, CYP2B6, CYP2C8, CYP2C19 and CYP3A4 as well as glucuronyl transferases and epoxide hydrolase [28], some of which are involved in the metabolism of ABZ (CYP3A4) and PZQ (CYP2B1 and CYP3A4). Thus, patients taking carbamazepine or phenytoin may metabolize $\mathrm{ABZ}$ and $\mathrm{PZQ}$ at a faster rate, contributing to increased serum concentrations for $A B S O$ and decreased concentrations for PZQ.

In our study we also evaluated the kinetic disposition of PZQ during ABZ administration in NCC patients concomitantly treated with carbamazepine or phenytoin. PZQ is metabolized by CYP2B1 and CYP3A4 [29]. Higher plasma PZQ concentrations were found in patients treated with phenytoin than in those treated with carbamazepine, probably because of carbamazepine-associated CYP3A4 induction [29]. Bittencourt et al. [30] reported that both carbamazepine and phenytoin decreased plasma concentrations of PZQ because of increased clearance secondary to induction of first-pass liver metabolism.

In another, single-dose study in healthy volunteers, combined $A B Z$ and $P Z Q$ increased plasma concentrations of (+)-ABSO, (-)-ABSO, ASON and (-)-(R)-PZQ [31]. The authors suggested that $P Z Q$ could function as an inhibitor of intestinal P-glycoprotein with ABSO as its substrate considering that enzymatic induction does not occur as a result of the administration of $P Z Q$ as a single dose.

Combined use of $A B Z+P Z Q$ was associated with higher concentrations of $A B S O$ and absence of additional side effects in this small series. This information, added to early efficacy data from controlled studies [15], suggests that combined $A B Z+P Z Q$ therapy may provide a suitable alternative for the treatment of NCC. Precise identification of its phamacokinetics in the context of other medications commonly used for the treatment of NCC should help to design sound therapeutic regimens and avoid treatment failures secondary to drug interactions.

\section{Competing Interests}

The authors have no competing interests to declare.

\section{Funding}

This study was funded by the National Institute of Neurological Diseases and Stroke, National Institutes of Health, USA, through grant R01 054805. Support from Training Grant TW001140 from the Fogarty International Center in training of study team members is also acknowledged.

Hector H. Garcia is now a Wellcome Trust International Senior Research Fellow in Public Health.

Drs Antonio Delgado-Escueta, Marco T.Medina and Oscar Del Brutto provided valuable methodological and conceptual advice. Drs M. Alvarado, M. Porras, V. Vargas and A. Cjuno contributedt to patient referral and neurological care. We are very grateful to the team of study coordinators (A. Chuquichanca, J. Del Carpio, K. Fernandez) and to our laboratory personnel who processed all samples ( $Y$. Castillo). Comments and suggestions from the study DSMB and sponsoring officers greatly contributed to improve the study protocol and performance.

\section{REFERENCES}

1 Commission on Tropical Diseases of the International League Against Epilepsy. Relationship between epilepsy and tropical diseases. Commission on Tropical Diseases of the International League Against Epilepsy. Epilepsia 1994; 35: 89-93.

2 Garcia HH, Pretell EJ, Gilman RH, Martinez SM, Moulton LH, Del Brutto OH, Herrera G, Evans CA, Gonzalez AE. A trial of antiparasitic treatment to reduce the rate of seizures due to cerebral cysticercosis. N Engl J Med 2004; 350: 249-58.

3 Del Brutto OH, Roos KL, Coffey CS, Garcia HH. Meta-analysis: cysticidal drugs for neurocysticercosis: albendazole and praziquantel. Ann Intern Med 2006; 145: 43-51. 
4 Sotelo J, del Brutto OH, Penagos P, Escobedo F, Torres B, Rodriguez-Carbajal J, Rubio-Donnadieu F. Comparison of therapeutic regimen of anticysticercal drugs for parenchymal brain cysticercosis. J Neurol 1990; 237:69-72.

5 Homeida M, Leahy W, Copeland S, Ali MM, Harron DW. Pharmacokinetic interaction between praziquantel and albendazole in Sudanese men. Ann Trop Med Parasitol 1994; 88: 551-9.

6 Pengsaa K, Na-Bangchang K, Limkittikul K, Kabkaew K, Lapphra K, Sirivichayakul C, Wisetsing P, Pojjaroen-Anant C, Chanthavanich P, Subchareon A. Pharmacokinetic investigation of albendazole and praziquantel in Thai children infected with Giardia intestinalis. Ann Trop Med Parasitol 2004; 98: 349-57.

7 Na-Bangchang K, Kietinun S, Pawa KK, Hanpitakpong W, Na-Bangchang C, Lazdins J. Assessments of pharmacokinetic drug interactions and tolerability of albendazole, praziquantel and ivermectin combinations. Trans $\mathrm{R}$ Soc Trop Med Hyg 2006; 100: 335-45.

8 Taylor DH, Morris DL. Combination chemotherapy is more effective in postspillage prophylaxis for hydatid disease than either albendazole or praziquantel alone. Br J Surg 1989; 76: 954.

9 Ayles HM, Corbett EL, Taylor I, Cowie AG, Bligh J, Walmsley K, Bryceson AD. A combined medical and surgical approach to hydatid disease: 12 years' experience at the Hospital for Tropical Diseases, London. Ann R Coll Surg Engl 2002; 84: 100-5.

10 Lam KS, Faraj A, Mulholland RC, Finch RG. Medical decompression of vertebral hydatidosis. Spine 1997; 22: 2050-5.

11 Chawla A, Maheshwari M, Parmar H, Hira P, Hanchate V. Imaging features of disseminated peritoneal hydatidosis before and after medical treatment. Clin Radiol 2003; 58: 818-20.

12 Miquel J, Gil-Grande LA, Retamar A, Garcia-Hoz F, del Pozo D, Pena E. [Response of multiorganic hydatdosis to combined therapy with albendazole and praziquantel]. Enferm Infecc Microbiol Clin 2001; 19: 139-40.

13 Schaub NA, Perruchoud AP, Buechner SA. Cutaneous larva migrans associated with Loffler's syndrome. Dermatology 2002; 205: 207-9.

14 Li S, He G, Lu Z, Li Z, Tao Y, Zhang L, Zhu F. [Efficacy of praziquantel combined with albendazole in the treatment of clonorchiasis]. Zhongguo Ji Sheng Chong Xue Yu Ji Sheng Chong Bing Za Zhi 1995; 13:61-3.

15 Kaur S, Singhi P, Singhi S, Khandelwal N. Combination therapy with albendazole and praziquantel versus albendazole alone in children with seizures and single lesion neurocysticercosis: a randomized, placebo-controlled double blind trial. Pediatr Infect Dis J 2009; 28: 403-6.

16 Guo DM, Xie SP, Jia JP. [Therapeutic efficacy of praziquantel, albendazole and a combination of the two drugs in cysticercosis]. Zhongguo Ji Sheng Chong Xue Yu Ji Sheng Chong Bing Za Zhi 2003; 21: 187-8.
17 Venkatesan P. Albendazole. J Antimicrob Chemother 1998; 41: 145-7.

18 Mares SS, Jung CH, López AT, González-Esquivel DF. Influence of a Mexican diet on the bioavailability of albendazole. Basic Clin Pharmacol Toxicol 2005; 97: 122-4.

19 Castro N, Medina R, Sotelo J, Jung H. Bioavailability of praziquantel increases with concomitant administration of food. Antimicrob Agents Chemother 2000; 44: 2903-4.

20 Bonato PS, de Oliveira AR, de Santana FJ, Fernandes BJ, Lanchote VL, Gonzalez AE, Garcia HH, Takayanagui OM. Simultaneous determination of albendazole metabolites, praziquantel and its metabolite in plasma by high-performance liquid chromatography-electrospray mass spectrometry. J Pharm Biomed Anal 2007; 44: 558-63.

21 Rawden HC, Kokwaro GO, Ward SA, Edwards G. Relative contribution of cytochromes P-450 and flavin-containing monoxygenases to the metabolism of albendazole by human liver microsomes. Br J Clin Pharmacol 2000; 49: 313-22.

22 Steiger U, Cotting J, Reichen J. Albendazole treatment of echinococcosis in humans: effects on microsomal metabolism and drug tolerance. Clin Pharmacol Ther 1990; 47: 347-53.

23 Gottschall DW, Theodorides VJ, Wang R. The metabolism of benzimidazole anthelmintics. Parasitol Today 1990; 6: 115-24.

24 Ridtitid W, Ratsamemonthon K, Mahatthanatrakul W, Wongnawa M. Pharmacokinetic interaction between ketoconazole and praziquantel in healthy volunteers. J Clin Pharm Ther 2007; 32: 585-93.

25 Masimirembwa CM, Hasler JA. Characterisation of praziquantel metabolism by rat liver microsomes using cytochrome P450 inhibitors. Biochem Pharmacol 1994; 48: 1779-83.

26 Ridtitid W, Wongnawa M, Mahatthanatrakul W, Punyo J, Sunbhanich M. Rifampin markedly decreases plasma concentrations of praziquantel in healthy volunteers. Clin Pharmacol Ther 2002; 72: 505-13.

27 Castro N, Márquez-Caraveo C, Brundage RC, González-Esquivel D, Suárez AM, Góngora F, Jara A, Urizar J, Lanao JM, Jung $\mathrm{H}$. Population pharmacokinetics of albendazole in patients with neurocysticercosis. Int J Clin Pharmacol Ther 2009; 47:679-85.

28 Yap KY, Chui WK, Chan A. Drug interactions between chemotherapeutic regimens and antiepileptics. Clin Ther 2008; 30: 1385-407.

29 Perucca E. Clinically relevant drug interactions with antiepileptic drugs. Br J Clin Pharmacol 2006; 61:246-55.

30 Bittencourt PRM, Gracia CM, Martins R, Fernandes AG, Diekmann HW, Jung W. Phenytoin and carbamazepine decrease oral bioavailability of praziquantel. Neurology 1992; 42: 492-6.

31 Lima RM, Ferreira MA, de Jesus Ponte Carvalho TM, Dumêt Fernandes BJ, Takayanagui OM, Garcia HH, Coelho EB, Lanchote VL. Albendazole-praziquantel interaction in healthy volunteers: kinetic disposition, metabolism and enantioselectivity. Br J Clin Pharmacol 2011; 71:528-35. 